\title{
ALPPS offers a better chance of complete resection in patients with primarily unresectable liver tumors compared with conventional-staged hepatectomies: results of a multicenter analysis
}

Schadde, Erik ; Ardiles, Victoria ; Slankamenac, Ksenija ; Tschuor, Christoph ; Sergeant, Gregory ; Amacker, Nadja ; Baumgart, Janine ; Croome, Kris ; Hernandez-Alejandro, Roberto ; Lang, Hauke ; de Santibaňes, Eduardo ; Clavien, Pierre-Alain

\begin{abstract}
BACKGROUND: Portal vein occlusion to increase the size of the future liver remnant (FLR) is well established, using portal vein ligation (PVL) or embolization (PVE) followed by resection 4-8 weeks later. Associating liver partition with portal vein ligation for staged hepatectomy (ALPPS) combines PVL and complete parenchymal transection, followed by hepatectomy within 1-2 weeks. ALPPS has been recently introduced but remains controversial. We compare the ability of ALPPS versus PVE or PVL for complete tumor resection. METHODS: A retrospective review of all patients undergoing ALPPS or conventional staged hepatectomies using PVL or PVE at four high-volume HPB centres between 2003 and 2012 was performed. Patients with primary liver tumors and liver metastases were included. Primary endpoint was complete tumor resection. Secondary endpoints include 90-day mortality, complications, FLR increase, time to resection, and tumor recurrence. RESULTS: Forty-eight patients with ALPPS were compared with 83 patients with conventional-staged hepatectomies. Eighty-three percent (40/48 patients) of ALPPS patients achieved complete resection compared with $66 \%$ (55/83 patients) in PVE/PVL (odds ratio 3.34, $\mathrm{p}=0.027)$. Ninety-day mortality in ALPPS and PVE/PVL was 15 and $6 \%$, respectively (p $=0.2$ ). Extrapolated growth rate was 11 times higher in ALPPS (34.8 cc/day; interquartile range (IQR) 26-49) compared with PVE/PVL (3 cc/day; IQR2-6; $\mathrm{p}=0.001)$. Tumor recurrence at 1 year was 54 versus $52 \%$ for ALPPS and PVE/PVL, respectively $(\mathrm{p}=0.7)$. CONCLUSIONS: This study provides evidence that ALPPS offers a better chance of complete resection in patients with primarily unresectable liver tumors at the cost of a high mortality. The technique is promising but should currently not be used outside of studies and registries.
\end{abstract}

DOI: https://doi.org/10.1007/s00268-014-2513-3

Posted at the Zurich Open Repository and Archive, University of Zurich

ZORA URL: https://doi.org/10.5167/uzh-98976

Journal Article

Published Version

Originally published at:

Schadde, Erik; Ardiles, Victoria; Slankamenac, Ksenija; Tschuor, Christoph; Sergeant, Gregory; Amacker, Nadja; Baumgart, Janine; Croome, Kris; Hernandez-Alejandro, Roberto; Lang, Hauke; de Santibaňes, Eduardo; Clavien, Pierre-Alain (2014). ALPPS offers a better chance of complete resection in patients with primarily unresectable liver tumors compared with conventional-staged hepatectomies: results of a multicenter analysis. World Journal of Surgery, 38(6):1510-1519. 
DOI: https://doi.org/10.1007/s00268-014-2513-3 


\title{
ALPPS Offers a Better Chance of Complete Resection in Patients with Primarily Unresectable Liver Tumors Compared with Conventional-Staged Hepatectomies: Results of a Multicenter Analysis
}

\author{
Erik Schadde Victoria Ardiles $\cdot$ Ksenija Slankamenac $\cdot$ Christoph Tschuor \\ Gregory Sergeant • Nadja Amacker • Janine Baumgart • Kris Croome • \\ Roberto Hernandez-Alejandro • Hauke Lang • Eduardo de Santibaňes • \\ Pierre-Alain Clavien
}

Published online: 19 April 2014

(C) Société Internationale de Chirurgie 2014

\begin{abstract}
Background Portal vein occlusion to increase the size of the future liver remnant (FLR) is well established, using portal vein ligation (PVL) or embolization (PVE) followed by resection 4-8 weeks later. Associating liver partition with portal vein ligation for staged hepatectomy (ALPPS) combines PVL and complete parenchymal transection, followed by hepatectomy within 1-2 weeks. ALPPS has been recently introduced but remains controversial. We compare the ability of ALPPS versus PVE or PVL for complete tumor resection.

Methods A retrospective review of all patients undergoing ALPPS or conventional staged hepatectomies using PVL or PVE at four high-volume HPB centres between 2003 and 2012 was performed. Patients with primary liver
\end{abstract}

E. Schadde - K. Slankamenac - C. Tschuor - G. Sergeant .

P.-A. Clavien $(\varangle)$

Department of Surgery and Transplantation, Swiss HPB Center,

University Hospital Zurich, Rämistrasse 100, 8091 Zurich,

Switzerland

e-mail: clavien@access.usz.ch

V. Ardiles · E. de Santibaňes

Division of HPB Surgery and Liver Transplant Unit, Department of Surgery, Italian Hospital, Buenos Aires, Argentina

N. Amacker

Department of Radiology, University Hospital Zurich, Zurich, Switzerland

J. Baumgart $\cdot$ H. Lang

Department of Visceral Surgery and Transplantation, University of Mainz, Mainz, Germany

K. Croome $\cdot$ R. Hernandez-Alejandro

Division of HPB Surgery, Department of Surgery, Western

University, London, ON, Canada tumors and liver metastases were included. Primary endpoint was complete tumor resection. Secondary endpoints include 90-day mortality, complications, FLR increase, time to resection, and tumor recurrence.

Results Forty-eight patients with ALPPS were compared with 83 patients with conventional-staged hepatectomies. Eighty-three percent (40/48 patients) of ALPPS patients achieved complete resection compared with $66 \%(55 / 83$ patients) in PVE/PVL (odds ratio 3.34, $p=0.027$ ). Ninetyday mortality in ALPPS and PVE/PVL was 15 and $6 \%$, respectively $(p=0.2)$. Extrapolated growth rate was 11 times higher in ALPPS ( $34.8 \mathrm{cc} / \mathrm{day}$; interquartile range (IQR) 26-49) compared with PVE/PVL (3 cc/day; IQR2-6; $p=0.001)$. Tumor recurrence at 1 year was 54 versus $52 \%$ for ALPPS and PVE/PVL, respectively $(p=0.7)$.

Conclusions This study provides evidence that ALPPS offers a better chance of complete resection in patients with primarily unresectable liver tumors at the cost of a high mortality. The technique is promising but should currently not be used outside of studies and registries.

\section{Introduction}

Resection of a large tumor load in the liver may result in an excessive removal of hepatic parenchyma leading to postoperative liver failure and associated complications [1]. This has led to the use of portal vein manipulations to increase the size of the putative future liver remnant (FLR). Multiple modifications have been described including a variety of two-stage surgeries combining removal of tumors from the FLR with portal vein embolization (PVE) $[2,3]$ or concomitant portal vein ligation $(\mathrm{PVL})[1,4]$ as well as PVE followed by extended right hepatectomy. 
A new variant of portal vein occlusion associated with staged hepatectomy was recently described to enhance volume increase of the FLR [5]. This approach combines liver partition with PVL followed by a second operation to remove the deportalized, diseased part of the liver. The acronym "ALPPS" (Associating Liver Partition with Portal Vein Ligation for Staged Hepatectomy) has been proposed to describe this complex procedure [6]. Initial experience suggested that the volume increase after ALPPS is more rapid compared with previous techniques allowing removal of the diseased part of the liver within only 1-2 weeks after liver partition [5, 7]. Several other groups have subsequently described the feasibility of ALPPS [8$10]$, and the procedure was rapidly implemented by many to attempt curative liver resection in patients with small FLRs. Despite its potential to induce rapid volume increase, ALPPS may be associated with higher postoperative morbidity and mortality rates $[5,8]$. An editorial and several letters to the Annals of Surgery have sparked a controversy over the benefits and dangers of the ALPPS procedure [11-17].

Both PVE and PVL carry a considerable failure rate, because only about two thirds of patients may eventually benefit from a subsequent curative resection due to tumor progression during the waiting interval between the two stages or failure of the FLR to grow [2, 4, 18-20]. While some consider tumor progression in the waiting interval as a useful selection tool to avoid an extensive liver resection in patients with unfavourable tumor biology [14], others hypothesized that the long time interval between the two stages rather than tumor biology is responsible for the high degree of disease progression between stages [5-9, 21]. Recently, proponents of PVE have compared their own results with the inaugural German series to argue against the innovation [22]. Conclusive evaluations of overall and disease-free survival comparing the two techniques will require large patient populations, which are currently not yet available. The purpose of this study therefore was to compare the ability of ALPPS versus conventional two-stage approaches (using PVL or PVE) to achieve complete tumor resection using a short-time endpoint instead, allowing for appropriate sample size and avoidance of single-centre bias by using a multicenter design.

\section{Materials and methods}

Primary endpoint

The primary endpoint was reached when the second stage was performed with resection of the entire tumor load with free margins in the pathology specimen. The sample size of patients necessary to answer the question whether ALLPS was better than PVE/PVL to achieve complete tumor resection was based on literature data suggesting nonprogression to the second stage and thereby failure of the entire strategy in up to a third of patients in PVE/PVL [2, 4, $8,18,19]$ and in nearly no patients for ALPPS [5, 7]. Assuming a power of 0.8 and $\alpha$-error of 0.05 , more than 40 patients were needed in each arm. Therefore, four international centers (Zurich, Switzerland; London, Ontario, Canada; Buenos Aires, Argentina; Mainz, Germany) with experience with the ALPPS procedure collaborated to pool more than 40 consecutive ALPPS and PVE/PVL patients in each arm. Patients who failed the primary endpoint were classified according to four patterns of failure: (A) perioperative death, (B) no stage 2 because of tumor progression, (C) no stage 2 because of failure to grow, (D) incomplete resection $(\mathrm{R} 1)$.

\section{Patients}

All consecutive patients, who underwent ALPPS performed between January 2011 and September 2012 in the four collaborating centres, were compared with all patients who underwent conventional approaches (PVE/ PVL) performed between January 2003 and September 2012 in the same centres. Patients presenting with major extrahepatic surgery or subjected to selective intra-arterial chemotherapy and those with incomplete data on liver volumetry or lost to follow-up were excluded. An institutional review board (IRB) approval was obtained in each center.

\section{Surgical technique}

The surgical technique for ALPPS and conventional twostage liver resections associated with PVE or PVL have been described elsewhere [5, 7]. In brief, for ALPPS, stage 1 consists of tumor clearance of the FLR in case of multifocal bi-lobar tumors followed by parenchymal transection between the FLR and the diseased part of the liver with concomitant selective PVL. In cases of single large central tumors, transection with PVL is performed only.

In the PVL group, the FLR is cleared of tumor and the portal vein to the diseased hemi-liver is ligated during the first stage without concomitant parenchymal transection, in contrast to ALPPS. In the PVE group, patients undergo percutaneous PVE with coils or histoacryl/lipiodol, either alone (in case of unilobar disease) or 1-2 weeks after tumor clearance of the FLR in patients with multifocal bilobar tumors. In PVE and PVL, the diseased deportalized part of the liver is removed 4-8 weeks later. 


\section{Liver volumetry}

For all groups, baseline FLR volume (FLR1, i.e., before stage 1) and volume before stage 2 (FLR2) were measured by computer tomography (CT) or magnetic resonance imaging (MRI) using dedicated volume rendering software $[23,24]$. To standardize the speed of volume increase between the two groups, a mean volume increase per day was calculated. Since time intervals between stages differed between ALPPS and PVE/PVL, this assessment of kinetic growth was considered an approximation. Standardized total liver volume (sTLV) was calculated according to Vauthey [25]. The Mosteller formula was used to calculate body surface area. Standardized FLR1 (sFLR1) and sFLR2 were calculated accordingly as FLR1/ sTLV*100 \% and FLR2/sTLV*100 \%, respectively.

\section{Secondary endpoints}

Secondary endpoints included: 90-day mortality, overall and severe complications, comprehensive complication index (CCI) [26], postoperative liver and renal failure, and tumor recurrence up to 12 months. The study was not powered to detect differences in secondary endpoints. Complications were recorded using the Clavien-Dindo classification [27]; a severe complication was defined as grade $\geq$ IIIB (requirement of invasive procedures under general anesthesia to correct a complication). The novel CCI was reported to summarize for the first time all postoperative complications and their severities over both stages into one single continuous scale (www.assessurgery. com) [26]. Postoperative liver failure was defined according to the 50/50 criteria [28], renal failure as an increase of creatinine within $48 \mathrm{~h}$ after surgery to more than 1.4 of the preoperative level [29]. Tumor progression and recurrence were assessed up to 12 months starting to count from the first stage in both arms.

American Society of Anesthesiologists (ASA) physical status classification system was coded based on the definition provided on the ASA webpage (www.asahq.org). Charlson score was determined using a Microsoft ${ }^{\circledR}$ Excel macro [30]. Type of tumor and histology was coded based on pathology source documents. Tumor size and number of lesions were defined through primary review of imaging by experienced radiologists in each center.

\section{Statistical analysis}

The distribution of variables was analyzed using means and standard deviation (SD) for normally distributed, and median and interquartile ranges (IQR) for nonnormally distributed data. Data were tested for normality using quantile-quantile plots of dependent variables.
The primary endpoint (complete resection with RO margins) was compared between the two groups (ALPPS vs. PVE/PVL) using uni- and multivariate logistic regression models with the primary endpoint as the dependent and treatment group as the independent variable. We adjusted for following potential confounders: age, previous abdominal surgery (yes/no), type of tumor, FLR1/body weight (BW), and liver macrosteatosis (yes/no). Uni- and multivariate linear as well as logistic regression analyses were performed for the secondary endpoints. Data were reported as point estimates, $95 \%$ confidence intervals (CI), and $p$ values $(\leq 0.05$ considered as significant).

Kaplan-Meier survival curves were constructed for time to proceed to the second stage and progression free survival. Associating liver partition with portal vein ligation for staged hepatectomy and PVE/PVL were compared using log-rank statistics.

All statistical analyses were performed using STATA 11 (Stata Corp., College Station, TX). Figures were made using Graph Pad Prism (Graph Pad Software, La Jolla, CA).

\section{Results}

A total of 170 patients with liver tumors undergoing liver resections in two stages at the four centres were analyzed. Thirty-nine patients were excluded because of simultaneous extrahepatic surgery $(n=4)$, placement of selective intra-arterial perfusion pumps $(n=14)$, had benign tumors $(n=7)$, had no appropriate imaging $(n=13)$, or were lost to follow-up within 3 months $(n=1)$. A total of 131 patients were eventually included: 48 with ALPPS and 83 with PVE/PVL (Fig. 1).

Comparison of patient population demographics, morbidity, and comorbidity data showed a higher percentage of mild hepatic macrosteatosis $(<30 \%)$ in the ALPPS group as well as difference in the number of enrolled patients per centre (Table 1). Future liver remnant in cubic centimetres and sFLR in percent before stage 1 (FLR1 and sFLR1), as well FLR to body weight ratio before stage 1 (FLR1/BW) were not statistically different between both groups. Need for biliary reconstruction was higher in ALPPS in stage 1 and higher in PVE/PVL in stage 2, but in both stages, comparable (Table 2).

Eighty-three percent (40/48) of ALPPS patients achieved complete resection compared with $66 \%$ (55/83) in the PVE/ PVL group. Seventeen percent (8/48) of ALPPS patients failed the primary endpoint due to (A) mortalities $(n=7)$ and due to one patient with (D) incomplete resection (R1) (Table 3).

In comparison, 28 of 83 patients ( $34 \%$ ) in the PVE/PVL group did not reach complete resection because of 
Fig. 1 Flowchart of patients screened and included in the study

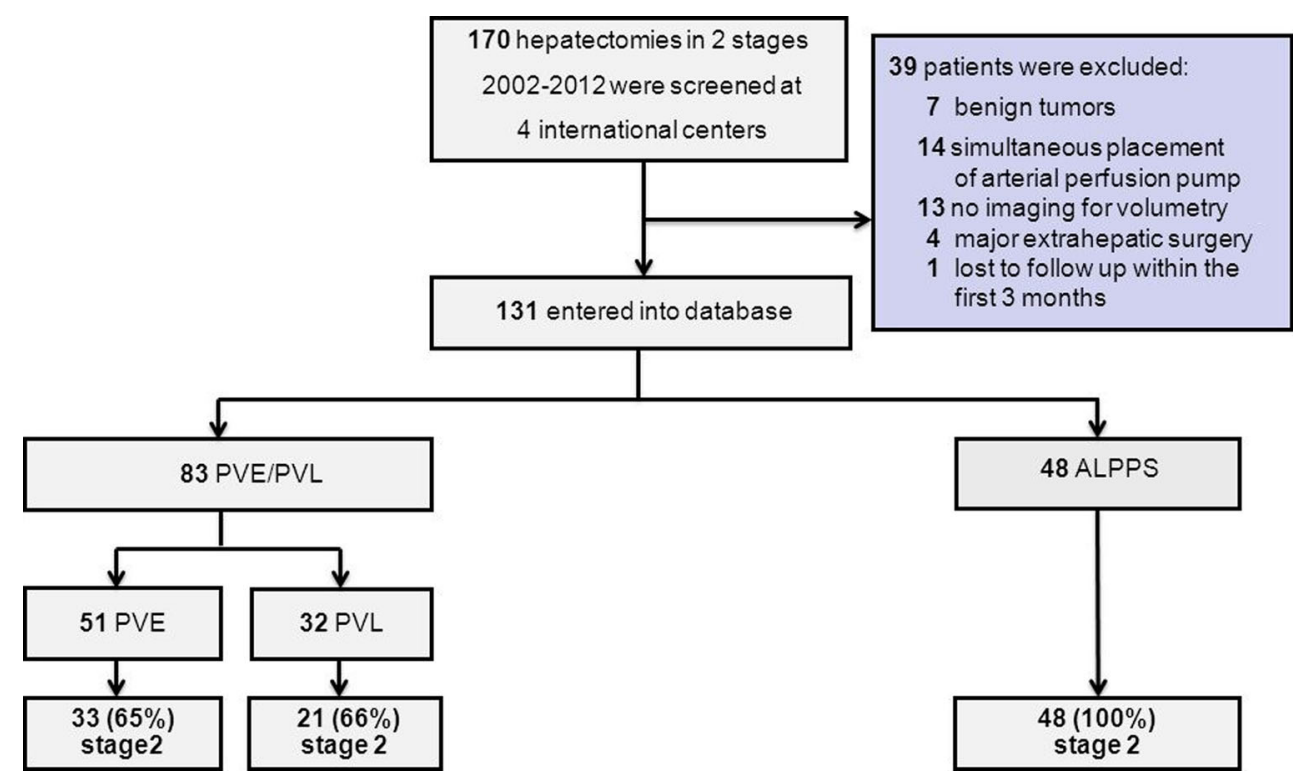

(A) postoperative mortality $(n=5)$, (B) liver $(n=4)$ or systemic $(n=9)$ tumor progression, (C) failure of the FLR to grow $(n=6)$ and $\mathrm{R} 1$ resection in 4 patients (Table 3 ). Results of the multivariate analysis for primary and secondary endpoints and the odds ratios of patients with ALPPS for these endpoints, unadjusted and adjusted for age, previous abdominal surgery, type of tumor, FLR1/BW, and liver macrosteatosis are shown in Table 4; ALPPS was more likely to achieve complete resection (adjusted OR 3.34, CI 1.15-9.74, $p=0.027$ ).

Mortality at 90 days was $15 \%(7 / 48)$ in ALPPS compared with $6 \%(5 / 83)$ in PVE/PVL, i.e., the corrected odds for perioperative death were 2.7 time higher $(p=0.2$; Table 4). Severe complications were more common in ALPPS after both steps compared with PVE/PVL, but the numbers were too small to show significance. In both groups, liver failure occurred only after stage 2 at 13 and $9 \%$ in ALPPS and PVE/PVL, respectively. There was a trend towards more overall complications in the ALPPS group according to the new CCI $(p=0.05)$. There were no differences in the incidence of postoperative bile leaks. The incidence of acute renal failure after stage 1 in ALPPS seemed high at $8 \%(4 / 48)$, but renal failure after stage 2 was not different between groups at 10 and $15 \%$ for ALLPS and PVE/PVL, respectively (Table 4).

Median FLR2, sFLR2, and FLR2/BW (i.e., before stage 2) were higher in ALPPS than in PVE/PVL (Table 5). Each ALPPS patient reached the $30 \%$ sFLR cutoff recommended for safer liver surgery [1] before stage 2, whereas many PVE/PVL cases did not (Fig. 2). Increase of FLR volume between stage 1 and 2 in both groups was significant $(p \leq 0.001)$ (Fig. 2). The median increase of FLR between stages was $34 \%$ for PVE/PVL and $77 \%$ for
ALLPS (Table 5). Extrapolated kinetic growth for ALPPS was 11 times higher (34.8 cc/day, IQR 26.4-48.5) compared with PVE/PVL (2.78 cc/day, IQR 1.69-5.81; Fig. 2; Table 5).

Whereas ALPPS patients proceeded to resection faster (Fig. 3a), tumor recurrence occurred at a comparable rate in both groups at 12 months with $54 \%$ in ALPPS and $52 \%$ in PVE/PVL (Fig. 3b).

\section{Discussion}

Patients with primarily unresectable liver tumors have a poor prognosis with a near zero 5-year survival despite the availability of modern chemotherapy. Because only a curative resection offers a chance of long-term survival, strategies using staged hepatectomies have been developed over the past two decades, however, with limited success [2, 4, 18, 19]. It has been suggested that the recently introduced ALPPS procedure offers new horizons to remove extensive tumors localized to the liver by stimulating regeneration of the healthy part of the liver at an unprecedented pace and extent $[5,6]$. This enthusiasm has been challenged by others due to lack of convincing data and fear of an increased rate of perioperative complications [12, 14].

While definitive evidence for a long-term benefit in survival of ALPPS will be lacking for a long time due to the large numbers of patients necessary to show a difference, it is critical to evaluate this approach before it is widely used or abandoned without objectively weighing its merits. While an attempt has been made to compare PVE to the published data about ALPPS [22], no large comparative study is currently 
Table 1 Characteristics of patients with unresectable liver tumors cohorts undergoing PVE/PVL or ALPPS
All data are given in proportions or in medians with interquartile ranges (IQR)

$P V E / P V L$ portal vein embolization/portal vein ligation, $A L P P S$ associating liver partition with portal vein ligation for staged hepatectomy, ASA American Society of Anesthesiologists Physical Status Classification, BMI body mass index, INR international normalized ratio, CRLM colorectal liver metastasis, $H C C$ hepatocellular carcinoma, IHCC intrahepatic

cholangiocarcinoma, $\mathrm{PHCC}$ perihilar cholangiocarcinoma, SOS sinusoidal obstruction syndrome, $C A S H$

chemotherapy-associated steatohepatitis, $\mathrm{CH}$ Switzerland, $C A$ Canada, $A R$ Argentina, $G E$ Germany

\begin{tabular}{|c|c|c|c|}
\hline Characteristics & $\begin{array}{l}\text { PVE/PVL group } \\
\text { Stage 1: } n=83 \\
\text { Stage } 2: n=54\end{array}$ & $\begin{array}{l}\text { ALPPS group } \\
\text { In stage } 1: n=48 \\
\text { In stage } 2: n=48\end{array}$ & $p$ value \\
\hline Age (year) & $61(54-69)$ & $57(48.5-65)$ & 0.11 \\
\hline Sex, male/female & $57(68.7 \%) / 26(31.3 \%)$ & $29(60.4 \%) / 19(39.6 \%)$ & 0.34 \\
\hline ASA & & & 0.07 \\
\hline$\leq 2$ & $57(68.7 \%)$ & $40(83.3 \%)$ & \\
\hline$>2$ & $26(31.3 \%)$ & $8(16.7 \%)$ & \\
\hline Charlson index & $7(6-9)$ & $8(4-9)$ & 0.47 \\
\hline Diabetes mellitus & $11(13.3 \%)$ & $4(8.3 \%)$ & 0.4 \\
\hline Type of tumor & & & 0.68 \\
\hline CRLM & $48(57.8 \%)$ & $26(54.2 \%)$ & \\
\hline $\mathrm{HCC}$ & $7(8.4 \%)$ & $3(6.3 \%)$ & \\
\hline Biliary carcinoma & $16(19.3 \%)$ & $10(20.8 \%)$ & \\
\hline IHCC & $5(6 \%)$ & & \\
\hline PHCC & $11(13.2 \%)$ & $2(4.2 \%)$ & \\
\hline Other malignant tumors & $12(14.5 \%)$ & $7(14.6 \%)$ & \\
\hline $\operatorname{BMI}\left(\mathrm{kg} / \mathrm{m}^{2}\right)$ & $25.4(23.1-28.7)$ & $25.9(23.4-28.8)$ & 0.95 \\
\hline Preoperative chemotherapy & $44(53 \%)$ & $28(58.3 \%)$ & 0.56 \\
\hline Creatinine baseline $(\mu \mathrm{mol} / \mathrm{L})$ & $71(62-86)$ & $71(62-82.2)$ & 0.49 \\
\hline Bilirubin baseline $(\mu \mathrm{mol} / \mathrm{L})$ & $12(8-20)$ & $11(6.6-15.7)$ & 0.09 \\
\hline INR baseline & $1(1-1.1)$ & $1(1-1.1)$ & 0.52 \\
\hline \multicolumn{4}{|l|}{ Preoperative biliary drainage } \\
\hline In stage 1 & $11(13.3 \%)$ & $4(8.3 \%)$ & 0.44 \\
\hline In stage 2 & $12(22.2 \%)$ & $5(10.4 \%)$ & 0.27 \\
\hline Previous liver surgery & $16(19.3 \%)$ & $9(18.8 \%)$ & 0.94 \\
\hline Multifocal bilobar tumor & $56(67.5 \%)$ & $29(60.4 \%)$ & 0.42 \\
\hline \multicolumn{4}{|l|}{ Number of lesions } \\
\hline$<5$ & $50(60.2 \%)$ & $23(47.9 \%)$ & 0.09 \\
\hline$\geq 5$ & $29(34.9 \%)$ & $25(52.1 \%)$ & \\
\hline Missing & $4(4.9 \%)$ & $0 \%$ & \\
\hline \multicolumn{4}{|l|}{ Histology } \\
\hline No histology & $29(34.9)$ & - & \\
\hline Normal & $25(30.2 \%)$ & $17(35.4 \%)$ & 0.10 \\
\hline \multicolumn{4}{|l|}{ Macrosteatosis } \\
\hline$>30 \%$ & $8(9.6 \%)$ & $2(4.2 \%)$ & 0.026 \\
\hline$<30 \%$ & $17(20.5 \%)$ & $21(43.7 \%)$ & 0.35 \\
\hline Fibrosis & $4(4.8 \%)$ & $6(12.5 \%)$ & - \\
\hline SOS & $0 \%$ & $2(4.2 \%)$ & - \\
\hline CASH & $0 \%$ & $0 \%$ & \\
\hline \multicolumn{4}{|l|}{ Centers } \\
\hline Zurich, $\mathrm{CH}$ & $40(48.2 \%)$ & $18(37.5 \%)$ & 0.24 \\
\hline London Ontario, CA & $21(25.3 \%)$ & $5(10.4 \%)$ & \\
\hline Buenos Aires, AR & $12(14.5 \%)$ & $15(31.3 \%)$ & \\
\hline Mainz, GE & $10(12 \%)$ & $10(20.8 \%)$ & \\
\hline
\end{tabular}

available. At this point, centres have reported small case series, mostly with a focus on feasibility and technical variations, such as the description of a laparoscopic approach
[16], providing only anecdotal information. Therefore, we designed a study with the endpoint complete resection, which is a relevant and sufficiently powered, short-term 
Table 2 Operative characteristics of patients with unresectable liver tumors cohorts undergoing PVE/PVL or ALPPS

\begin{tabular}{|c|c|c|c|}
\hline Stage 1 & $\begin{array}{l}\text { PVE/PLV group } \\
n=83\end{array}$ & $\begin{array}{l}\text { ALPPS group } \\
n=48\end{array}$ & $p$ value \\
\hline \multicolumn{4}{|l|}{ Type of surgery } \\
\hline PVO & $83(100 \%)$ & & \\
\hline PVE & $51(62.4 \%)$ & & \\
\hline PVL & $32(38.6 \%)$ & & \\
\hline ALPPS & - & $48(100 \%)$ & \\
\hline Size of FLR1 (cc) & $389(324-470)$ & $367(286-440)$ & 0.10 \\
\hline Size of sFLR1 & $0.24(0.18-0.31)$ & $0.23(0.18-0.29)$ & 0.07 \\
\hline FLR1/BW (cc/kg) & $0.53(0.39-0.67)$ & $0.47(0.39-0.59)$ & 0.06 \\
\hline Cleaning of the FLR & $55(66 \%)$ & $28(58.3)$ & 0.27 \\
\hline Biliary reconstruction & $0(0 \%)$ & $8(16.7 \%)$ & $<0.001$ \\
\hline \multicolumn{4}{|l|}{ Hepaticojejunostomy } \\
\hline One duct & & 5 & \\
\hline Multiple ducts & & 3 & \\
\hline Stage 2 & $\begin{array}{l}\text { PVE/PVL group } \\
n=54\end{array}$ & $\begin{array}{l}\text { ALPPS group } \\
n=48\end{array}$ & $p$ value \\
\hline Size of FLR2 (cc) & $530(454-648)$ & $639(525-786)$ & 0.007 \\
\hline Size of sFLR2 & $0.35(0.27-0.45)$ & $0.41(0.34-0.47)$ & 0.003 \\
\hline FLR2/BW (cc/kg) & $0.73(0.56-0.96)$ & $0.84(0.73-0.99)$ & 0.005 \\
\hline Biliary reconstruction & $15(27.8 \%)$ & $4(8.3 \%)$ & 0.06 \\
\hline \multicolumn{4}{|l|}{ Hepaticojejunostomy } \\
\hline One duct & 9 & 2 & \\
\hline Multiple ducts & 6 & 2 & \\
\hline
\end{tabular}

All data are given in proportions or in medians with interquartile ranges (IQR)

$P V O$ portal vein occlusion, $P V E / P V L$ portal vein embolization, portal vein ligation, $A L P P S$ associating liver partition with portal vein ligation for staged hepatectomy, FLR1 future liver remnant volume prior to stage 1, FLR2 future liver remnant volume prior to stage 2, sFLR1 standardized future liver remnant prior to stage $1, s F L R 2$ standardized future liver remnant prior to stage $2, F L R 1 / B W$ future liver remnant to body weight ratio prior to stage $1, F L R 2 / B W$ future liver remnant to body weight ratio prior to stage 2

Table 3 Reasons for failure of the primary endpoint

\begin{tabular}{lll}
\hline Reason for failure & $\begin{array}{l}\text { PVE/PVL } \\
n=83\end{array}$ & $\begin{array}{l}\text { ALPPS } \\
n=48\end{array}$ \\
\hline A. Perioperative death (3 months) n (\%) & $5(6 \%)$ & $7(15 \%)$ \\
B. No stage 2 because of tumor progression n (\%) & $13(16 \%)$ & 0 \\
Liver n & 4 & 0 \\
Systemic n & 9 & 0 \\
C. No stage 2 because of failure to grow n (\%) & $6(7 \%)$ & 0 \\
D. Incomplete resection (R1) n (\%) & $4(5 \%)$ & $1(2 \%)$ \\
Patients who failed primary endpoint $\mathrm{n}(\%)$ & $28(34 \%)$ & $8(17 \%)$ \\
\hline
\end{tabular}

surgical endpoint. The only chance of cure for this high-risk population, which often is offered only palliative chemotherapy, is a complete extirpation of the tumor, which requires staged procedures combining PVE or PVL and major hepatectomy at a later stage. This endpoint was chosen because achieving early complete resection is the indisputable basis for long-term survival.
The putative advantage of ALPPS is a faster regeneration of the FLR, which enables surgeons to proceed with the second stage before the development of adhesions or tumor progression. This key feature of ALLPS has been questioned by a few proponents of PVE, who claimed that the volume increase observed in ALPPS is similar to what can be achieved after right PVE, particularly with the 
Table 4 Clinical outcomes of the two patient cohorts using logistic and linear regression analysis correcting for important confounders

\begin{tabular}{|c|c|c|c|c|}
\hline & $\begin{array}{l}\text { PVE/PVL group } \\
\text { In stage } 1: n=83 \\
\text { In stage } 2: n=54 \\
\text { (proportion) }\end{array}$ & $\begin{array}{l}\text { ALPPS group } \\
\text { In stage } 1: n=48 \\
\text { In stage } 1: n=48 \\
\text { (proportion) }\end{array}$ & $\begin{array}{l}\text { Unadjusted odds ratio } \\
\text { (95\% CI, } p \text { value) }\end{array}$ & $\begin{array}{l}\text { Adjusted odds ratio } \\
\text { (95\% CI, } p \text { value) }\end{array}$ \\
\hline \multicolumn{5}{|l|}{ Primary endpoint } \\
\hline Complete resection (R0) & $55(66.3 \%)$ & $40(83.3 \%)$ & $\begin{array}{l}2.55 \\
(1.05-6.17, p=0.039)\end{array}$ & $\begin{array}{l}3.34 \\
(1.15-9.74, p=0.027)\end{array}$ \\
\hline \multicolumn{5}{|l|}{ Secondary endpoints } \\
\hline In-hospital mortality after stage 1 & $0 \%$ & $0 \%$ & - & - \\
\hline In-hospital mortality after stage 2 & $2(3.7 \%)$ & $7(14.6 \%)$ & $\begin{array}{l}4.4 \\
(0.9-22.5, p=0.072)\end{array}$ & $\begin{array}{l}2.47 \\
(0.34-17.45, p=0.368)\end{array}$ \\
\hline 90-day mortality & $5(6 \%)$ & $7(14.6 \%)$ & $\begin{array}{l}2.66 \\
(0.8-8.9, p=0.112)\end{array}$ & $\begin{array}{l}2.65 \\
(0.6-11.9, p=0.201)\end{array}$ \\
\hline Any complication after stage 1 & $21(25.3 \%)$ & $21(43.8 \%)$ & $\begin{array}{l}2.3 \\
(1.08-4.89, p=0.031)\end{array}$ & $\begin{array}{l}2.16 \\
(0.86-5.46, p=0.103)\end{array}$ \\
\hline Any complication after stage 2 & $40(74.1 \%)$ & $35(72.9 \%)$ & $\begin{array}{l}0.95 \\
(0.39-2.29, p=0.907)\end{array}$ & $\begin{array}{l}0.64 \\
(0.22-1.84, p=0.407)\end{array}$ \\
\hline Severe complications $(\geq$ IIIB) after stage 1 & $2(2.4 \%)$ & $7(14.6 \%)$ & $\begin{array}{l}6.9 \\
(1.4-34.8, p=0.019)\end{array}$ & - \\
\hline \multirow[t]{2}{*}{ Severe complications ( $\geq$ IIIB) after stage 2} & $8(14.8 \%)$ & $13(27.1 \%)$ & $\begin{array}{l}2.13 \\
(0.8-5.7, p=0.131)\end{array}$ & $\begin{array}{l}2.0 \\
(0.6-6.5, p=0.238)\end{array}$ \\
\hline & $\begin{array}{l}\text { PVE/PVL group } \\
\text { In stage } 1: n=83 \\
\text { In stage } 2: n=54 \\
\text { Median (IQR) }\end{array}$ & $\begin{array}{l}\text { ALPPS group } \\
\text { In stage } 1: n=48 \\
\text { In stage } 2: n=48 \\
\text { Median (IQR) }\end{array}$ & $\begin{array}{l}\text { Unadjusted difference } \\
\text { (95\% CI, } p \text { value) }\end{array}$ & $\begin{array}{l}\text { Adjusted difference } \\
\text { (95\% CI, } p \text { value) }\end{array}$ \\
\hline \multirow[t]{2}{*}{$\begin{array}{l}\text { Comprehensive } \\
\text { complications index }(\mathrm{CCI}) \text { for both stages }\end{array}$} & $20.9(8.7-30.8)$ & $26.2(8.7-44.9)$ & $\begin{array}{l}10.9 \\
(-0.3 \text { to } 22.0, p=0.057)\end{array}$ & $\begin{array}{l}11.5 \\
(-0.2 \text { to } 23.3, p=0.054)\end{array}$ \\
\hline & $\begin{array}{l}\text { PVE/PVL group } \\
\text { In stage } 1: n=83 \\
\text { In stage } 2: n=54 \\
\text { (proportion) }\end{array}$ & $\begin{array}{l}\text { ALPPS group } \\
\text { In stage } 1: n=48 \\
\text { In stage } 1: n=48 \\
\text { (proportion) }\end{array}$ & $\begin{array}{l}\text { Unadjusted odds ratio } \\
\text { (95\% CI, } p \text { value) }\end{array}$ & $\begin{array}{l}\text { Adjusted odds ratio } \\
\text { ( } 95 \% \text { CI, } p \text { value) }\end{array}$ \\
\hline Postoperative liver failure after stage $1^{\mathrm{a}}$ & $0 \%$ & $0 \%$ & - & - \\
\hline Postoperative liver failure after stage $2^{\mathrm{a}}$ & $5(9.3 \%)$ & $6(12.5 \%)$ & $\begin{array}{l}1.3 \\
(0.4-4.9, p=0.651)\end{array}$ & $\begin{array}{l}1.1 \\
(0.2-4.5, p=0.934)\end{array}$ \\
\hline Bile leak after stage 1 & $2(2.4 \%)$ & $1(2.1 \%)$ & - & - \\
\hline Bile leak after stage 2 & $9(16.7 \%)$ & $10(20.8 \%)$ & $\begin{array}{l}1.3 \\
(0.5-3.6, p=0.59)\end{array}$ & $\begin{array}{l}1.3 \\
(0.4-4.0, p=0.685)\end{array}$ \\
\hline Acute kidney failure after stage 1 & $2(2.4 \%)$ & $4(8.3 \%)$ & - & - \\
\hline Acute kidney failure after stage 2 & $8(14.8 \%)$ & $5(10.4 \%)$ & $\begin{array}{l}0.7 \\
(0.2-2.2, p=0.508)\end{array}$ & $\begin{array}{l}0.35 \\
(0.1-1.8, p=0.2)\end{array}$ \\
\hline
\end{tabular}

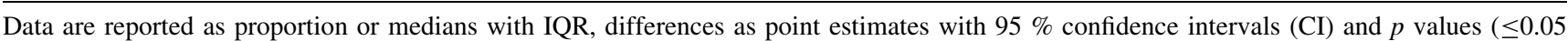
considered as significant)

Adjusted for age, previous abdominal surgery (non-liver), different diseases, FLR1/BW (prior to stage 1), and liver steatosis (yes/no). No statistical analysis if less than 5 patients

a By 50-50 criteria, see "Materials and methods" Section 
Table 5 Analysis of volume changes in patients undergoing ALPPS and PVE/PVL using linear regression analysis

\begin{tabular}{|c|c|c|c|c|}
\hline & $\begin{array}{l}\text { PVE/PVL group } \\
\text { In stage } 1: n=83 \\
\text { In stage } 2: n=54 \\
\text { Median (IQR) }\end{array}$ & $\begin{array}{l}\text { ALPPS group } \\
\text { In stage } 1: n=48 \\
\text { In stage } 2: n=48 \\
\text { Median (IQR) }\end{array}$ & $\begin{array}{l}\text { Unadjusted difference } \\
\text { (95\% CI, } p \text { value) }\end{array}$ & $\begin{array}{l}\text { Adjusted difference } \\
\text { (95\% CI, } p \text { value) }\end{array}$ \\
\hline FLR2 (before stage 2) (cc) & $530(454-648)$ & $638.5(525-785.5)$ & $\begin{array}{l}93.7 \\
(19.9-167.6, p=0.013)\end{array}$ & $\begin{array}{l}130.9 \\
(61.7-200.1, p<0.001)\end{array}$ \\
\hline sFLR2 (before stage 2) & $0.35(0.27-0.45)$ & $0.41(0.34-0.47)$ & $\begin{array}{l}0.05 \\
(0.01-0.09, p=0.019)\end{array}$ & $\begin{array}{l}0.08 \\
(0.05-0.11, p<0.001)\end{array}$ \\
\hline FLR2/BW ratio before stage $2(\mathrm{cc} / \mathrm{kg})$ & $0.73(0.56-0.96)$ & $0.84(0.73-0.99)$ & $\begin{array}{l}0.1 \\
(0.003-0.19, p=0.042)\end{array}$ & $\begin{array}{l}0.18 \\
(0.11-0.25, p<0.001)\end{array}$ \\
\hline Increase of FLR between stage 1 and $2(\%)$ & $34.1(17.4-55.7)$ & $77.4(52.8-101.7)$ & $\begin{array}{l}46.5 \\
(33.8-59.2, p<0.001)\end{array}$ & $\begin{array}{l}42 \\
(30.1-53.9, p<0.001)\end{array}$ \\
\hline Extrapolated kinetic growth (cc/day) & $2.78(1.69-5.81)$ & $34.8(26.4-48.5)$ & $\begin{array}{l}34.4 \\
(30.2-38.6, p<0.001)\end{array}$ & $\begin{array}{l}34 \\
(29.4-38.5, p<0.001)\end{array}$ \\
\hline
\end{tabular}

Data are reported as medians with IQR, differences as point estimates with $95 \%$ confidence intervals (CI) and $p$ values ( $\leq 0.05$ considered as significant) Adjusted for age, previous abdominal surgery (non-liver), different diseases, FLR1/BW (prior to stage 1) and liver steatosis (yes/no). All results are reported as median and interquartile range

$F L R 2$ future liver remnant prior to stage 2, sFLR2 standardized future liver remnant prior to stage 2, FLR2/BW ratio future liver remnant divided by body weight prior to stage 2

inclusion of segment four [14]. The results of this comparative analysis of 131 cases provide overwhelming evidence for a higher degree of liver regeneration with the ALPPS procedure, as previously suggested in small case series $[5,7,8]$. Of course, the limitation of our standardization of kinetic growth is the fact that is based on different time periods between stages as the denominator in ALPPS and PVE/PVL, shorter in ALPPS, longer in PVE/PVL. However, this study contributes to the evidence that rapid hypertrophy in ALPPS is real.

It has been challenged that reduced waiting time to proceed with complete resection may represent an oncological advantage. Several clinicians have argued that a long waiting interval in the conventional approaches is rather a selection tool to identify those who may best benefit from the completion hepatectomy [14]. Another concern raised was that livers are strongly manipulated in the ALPPS procedure, which may promote tumor cell dissemination by detaching cells into the systemic and pulmonary circulation. Additionally, the local and systemic release of growth factors may further stimulate tumor growth [14]. This study does not corroborate such concerns at least in the short-term, because recurrence occurred at a similar rate in both groups.

In the inaugural manuscript from Germany, ALPPS was successful in achieving complete resection at the cost of a postoperative mortality rate of $12 \%(3 / 25)$ [5], which is also in the range of the mortality observed in this study. While these figures are undoubtedly high, it is difficult to define what is acceptable mortality in a population presenting with such advanced malignancies and the potential

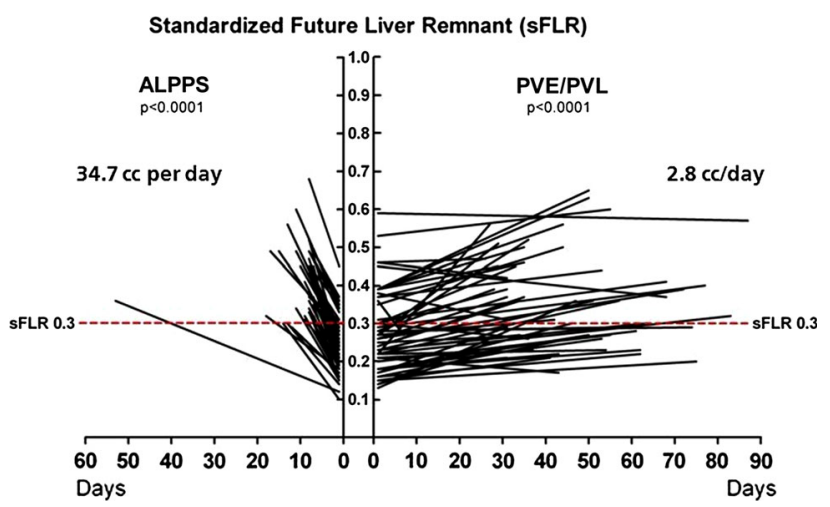

Fig. 2 Extrapolation of kinetic growth by depicting the increase of the standardized future liver remnant volumes (sFLR) determined by volumetry before stage 1 (middle) and before stage 2 in ALPPS (left) and PVE/PVL (right). The interrupted line shows the common clinical cutoff of $30 \%$ for safer liver surgery

for a curative surgical approach. Postoperative mortality has to be balanced with the risk of incomplete resection using the conventional approaches or in some cases the lack of alternative therapies in cases when the FLR is extremely small. Staged hepatectomies, including PVE or PVL, are associated with a postoperative mortality rate between 6 and $8 \%[2,4,18,19]$ compared with a 3-6\% mortality rate for conventional major hepatectomies [31]. The mortality of PVE/PVL in this series of 5 of $83(6 \%)$ is within the reported range. The 90-day mortality of $14.6 \%$ in our cohort represents the initial experience with the ALPPS operation and, without any doubt, includes our 


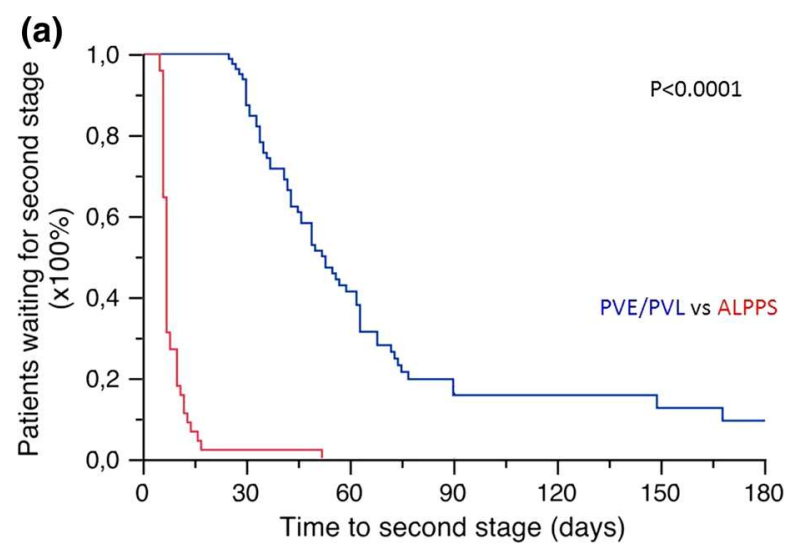

\begin{tabular}{|c|c|c|c|c|c|c|}
\hline 83 & 73 & 27 & 10 & 7 & 5 & 4 \\
\hline 48 & 2 & 0 & 0 & 0 & 0 & 0 \\
\hline
\end{tabular}

(b)

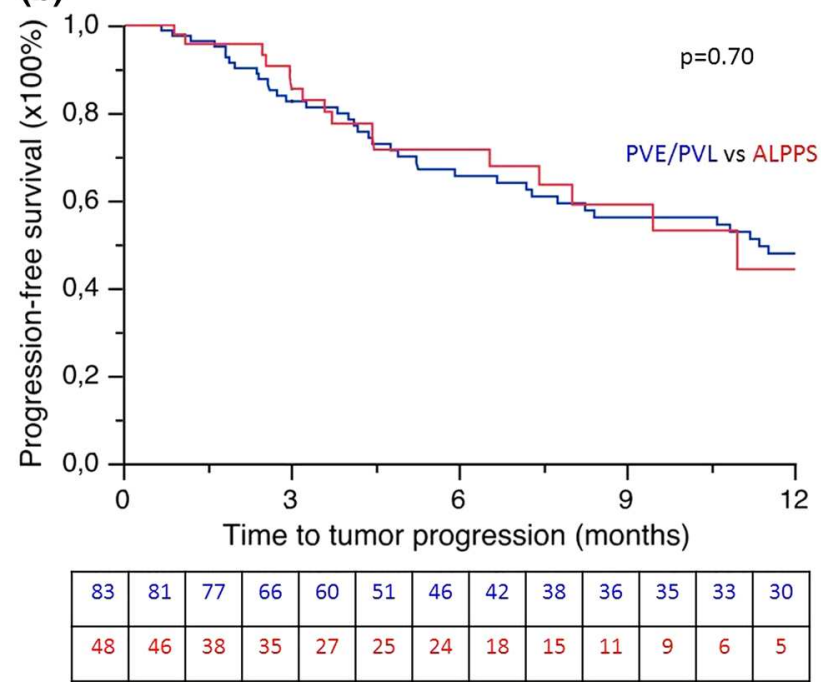

Fig. 3 a Kaplan-Meier graph demonstrating progression to stage 2 surgery comparing ALLPS with PVE/PVL. Numbers of patients at risk in the bottom line. b Kaplan-Meier graph demonstrating time to progression or recurrence or persistence of tumor after resection comparing ALLPS with PVE/PVL. Numbers of patients at risk in the bottom line

learning curve. In addition, this cohort inevitably has a selection bias, because patients may have been offered ALPPS because they were deemed inoperable by a conventional two-stage approach.

Liver failure, abdominal sepsis, and biliary leaks after surgery were the leading causes for severe complications and death in both groups, which is not different to what has been observed after conventional liver surgery [32]. Severe complications were rare after stage 1 in PVE/PVL, but occurred both after stage 1 and stage 2 in ALPPS. A risk factor analysis for complications failed to identify significant risk factors, probably due to the small sample size, although, of interest, five of seven fatalities in the ALPPS group occurred in patients older than 70 years of age and
Table 6 Recommendations for ALPPS

1. Best indication is a large tumor load with marginal future liver remnant (FLR) and curative intent

2. Should be used with caution in patients older than 70 years

3. Should be used with caution in patients with primary liver tumors (HCC, CCC)

4. Surgical team should have experience in complex liver surgery

5. Experience with in situ split or live donor liver transplantation might be of benefit

6. Avoid concomitant major abdominal surgery such as pancreatectomies and rectal resection

7. Informed consent mentioning higher perioperative morbidity and mortality should be obtained

8. Registration of patients in an international registry (www.alpps. net)

9. Should be preferentially performed in the setting of a prospective trial

five of seven fatalities occurred in patients with primary liver tumors. Considering the high morbidity and mortality, we caution the application of ALPPS as summarized in Table 6. With later knowledge and technical developments, revisions of these recommendations will become necessary.

This study is not without limitations. For example, the retrospective methodology yields a bias in the selection of patients in each group. ALPPS was chosen in an attempt to offer a curative operation to patients with extended liver malignancies, who had few options, but were interested in an aggressive, potentially curative, surgical approach. Also the time periods of patient inclusion differed, which is frequently unavoidable when new technologies are evaluated. However, we meticulously included all approaches involving the induction of liver hypertrophy by portal vein occlusion performed in the respective time periods at the respective centres. To address the concern about selection bias, we performed a multivariate analysis adjusting for known confounders. Secondly, the size of groups allowed us to evaluate our primary endpoint but did not provide enough power to convincingly address the differences in overall and disease-free survival, as well as morbidity. Finally, the majority of cases were performed very recently without sufficiently long follow-up to report long-term oncological results.

In conclusion, this study suggests that ALPPS offers a better chance of complete resection in patients with primarily unresectable liver tumors. This approach deserves further evaluation. Therefore, we implemented a registry (www.alpps.net) and initiated a multicenter RCT (www. clinicaltrials.gov; NCT 01775267). For the time being, due to the higher risk of morbidity and fatalities, we caution the widespread application of ALPPS outside of experienced centres. 
Acknowledgment This study was supported by the University of Zurich (Klinischer Forschungsschwerpunkt: Non-resectable Liver Tumors) and the Liver and Gastro-intestinal Disease Foundation (LGID) Swiss HPB Center, Zurich. We thank Sabine Kern, RN, and Lisette Paratore for their help in data collection for this manuscipt.

\section{References}

1. Clavien PA, Petrowsky H, DeOliveira ML et al (2007) Strategies for safer liver surgery and partial liver transplantation. N Engl J Med 356:1545-1559

2. Jaeck D, Oussoultzoglou E, Rosso E et al (2004) A two-stage hepatectomy procedure combined with portal vein embolization to achieve curative resection for initially unresectable multiple and bilobar colorectal liver metastases. Ann Surg 240:1037-1049 discussion 1049-1051

3. Adam R, Laurent A, Azoulay D et al (2000) Two-stage hepatectomy: a planned strategy to treat irresectable liver tumors. Ann Surg 232:777-785

4. Kianmanesh R, Farges O, Abdalla EK et al (2003) Right portal vein ligation: a new planned two-step all-surgical approach for complete resection of primary gastrointestinal tumors with multiple bilateral liver metastases. J Am Coll Surg 197:164-170

5. Schnitzbauer AA, Lang SA, Goessmann H et al (2012) Right portal vein ligation combined with in situ splitting induces rapid left lateral liver lobe hypertrophy enabling 2-staged extended right hepatic resection in small-for-size settings. Ann Surg 255:405-414

6. de Santibanes E, Clavien PA (2012) Playing Play-Doh to prevent postoperative liver failure: the "ALPPS" approach. Ann Surg 255:415-417

7. Alvarez FA, Ardiles V, Sanchez Claria R et al (2013) Associating liver partition and portal vein ligation for staged hepatectomy (ALPPS): tips and tricks. J Gastrointest Surg 17:814-821

8. Knoefel WT, Gabor I, Rehders A et al (2013) In situ liver transection with portal vein ligation for rapid growth of the future liver remnant in two-stage liver resection. Br J Surg 100:388-394

9. Li J, Girotti P, Konigsrainer I et al (2013) ALPPS in right trisectionectomy: a safe procedure to avoid postoperative liver failure? J Gastrointest Surg 17:956-961

10. Cavaness KM, Doyle MB, Lin Y et al (2013) Using ALPPS to induce rapid liver hypertrophy in a patient with hepatic fibrosis and portal vein thrombosis. J Gastrointest Surg 17:207-212

11. Clavien PA, Lillemoe KD (2012) Note from the editors on the ALPPS e-Letters-to-the-Editor. Ann Surg 256:552

12. Dokmak S, Belghiti J (2013) Which limits to the "ALPPS" approach? Ann Surg 256:e6 author reply e16-17

13. Narita M, Oussoultzoglou E, Ikai I et al (2012) Right portal vein ligation combined with in situ splitting induces rapid left lateral liver lobe hypertrophy enabling 2-staged extended right hepatic resection in small-for-size settings. Ann Surg 256:e7-e8 author reply e16-17

14. Aloia TA, Vauthey JN (2012) Associating liver partition and portal vein ligation for staged hepatectomy (ALPPS): what is gained and what is lost? Ann Surg 256:e9 author reply e16-19

15. Jain HA, Bharathy KG, Negi SS (2012) Associating liver partition and portal vein ligation for staged hepatectomy: will the morbidity of an additional surgery be outweighed by better patient outcomes in the long-term? Ann Surg 256:e10 author reply e16-17

16. Machado MA, Makdissi FF, Surjan RC (2013) ALPPS procedure with the use of pneumoperitoneum. Ann Surg Oncol 20(5):1491-1493

17. Andriani OC (2012) Long-term results with associating liver partition and portal vein ligation for staged hepatectomy (ALPPS). Ann Surg 256:e5 author reply e16-19

18. Tsai S, Marques HP, de Jong MC et al (2010) Two-stage strategy for patients with extensive bilateral colorectal liver metastases. HPB (Oxford) 12:262-269

19. Wicherts DA, Miller R, de Haas RJ et al (2008) Long-term results of two-stage hepatectomy for irresectable colorectal cancer liver metastases. Ann Surg 248:994-1005

20. Brouquet A, Abdalla EK, Kopetz S et al (2011) High survival rate after two-stage resection of advanced colorectal liver metastases: response-based selection and complete resection define outcome. J Clin Oncol 29:1083-1090

21. Sala S, Ardiles V, Ulla M et al (2012) Our initial experience with ALPPS technique: encouraging results. Updat Surg 64:167-172

22. Shindoh J, Vauthey JN, Zimmitti G et al (2013) Analysis of the efficacy of portal vein embolization for patients with extensive liver malignancy and very low future liver remnant volume, including a comparison with the associating liver partition with portal vein ligation for staged hepatectomy approach. J Am Coll Surg 217:126-133 discussion 133-134

23. van der Vorst JR, van Dam RM, van Stiphout RS et al (2010) Virtual liver resection and volumetric analysis of the future liver remnant using open source image processing software. World J Surg 34:2426-2433. doi:10.1007/s00268-010-0663-5

24. Dello SA, Stoot JH, van Stiphout RS et al (2011) Prospective volumetric assessment of the liver on a personal computer by nonradiologists prior to partial hepatectomy. World J Surg 35:386-392. doi:10.1007/s00268-010-0877-6

25. Vauthey JN, Abdalla EK, Doherty DA et al (2002) Body surface area and body weight predict total liver volume in Western adults. Liver Transpl 8:233-240

26. Slankamenac K, Graf R, Barkun J et al (2013) The comprehensive complication index: a novel continuous scale to measure surgical morbidity. Ann Surg 258:1-7

27. Dindo D, Demartines N, Clavien PA (2004) Classification of surgical complications: a new proposal with evaluation in a cohort of 6336 patients and results of a survey. Ann Surg 240:205-213

28. Balzan S, Belghiti J, Farges O et al (2005) The "50-50 criteria" on postoperative day 5: an accurate predictor of liver failure and death after hepatectomy. Ann Surg 242:824-828 discussion 828-829

29. Lameire N, Van Biesen W, Vanholder R (2008) Acute kidney injury. Lancet 372:1863-1865

30. Hall WH, Ramachandran R, Narayan S et al (2004) An electronic application for rapidly calculating Charlson comorbidity score. BMC Cancer 4:94

31. Asiyanbola B, Chang D, Gleisner AL et al (2008) Operative mortality after hepatic resection: are literature-based rates broadly applicable? J Gastrointest Surg 12:842-851

32. Breitenstein S, DeOliveira ML, Raptis DA et al (2010) Novel and simple preoperative score predicting complications after liver resection in noncirrhotic patients. Ann Surg 252:726-734 